\title{
Pericardial rupture with cardiac herniation following blunt thoracic trauma
}

\author{
Nicholas LeBlanc, MD, and Lawrence Tan, MD, Winnipeg, Manitoba, Canada
}

\footnotetext{
From the University of Manitoba-Winnipeg Health Sciences Centre, Winnipeg, Manitoba, Canada. Disclosures: The authors reported no conflicts of interest.

The Journal policy requires editors and reviewers to disclose conflicts of interest and to decline handling or reviewing manuscripts for which they may have a conflict of interest. The editors and reviewers of this article have no conflicts of interest.

Received for publication July 29, 2020; revisions received July 29, 2020; accepted for publication Aug 10, 2020; available ahead of print Aug 12, 2020.

Address for reprints: Nicholas LeBlanc, MD, 175 Emerite Dr, Lafayette, LA 70506 (E-mail: ndleblanc@att.net). JTCVS Techniques 2020;4:375-7

2666-2507

Published by Elsevier Inc. on behalf of The American Association for Thoracic Surgery. This is an open access article under the CC BY-NC-ND license (http://creativecommons.org/licenses/by-nc-nd/4.0/). https://doi.org/10.1016/j.xjtc.2020.08.011
}

$\rightarrow$ Video clip is available online.

A previously healthy 24-year-old male patient presented to a rural hospital with hemodynamic and respiratory instability following a high-speed motor vehicle collision involving multiple fatalities. He was managed with aggressive intravenous fluid resuscitation, blood transfusions, bilateral chest tubes, and intubation. Chest radiography revealed dextrocardia, and a focused assessment with sonography for trauma examination was limited secondary to massive subcutaneous emphysema and the heart's abnormal anatomical location. The patient was clinically stabilized with resuscitation, and a computed tomography angiogram of the chest was obtained which revealed cardiac herniation into the right pleural cavity with associated torsion, pulmonary artery and venous narrowing, bilateral hemopneumothoraces, pneumomediastinum, pulmonary contusions, and multiple left-sided rib fractures (Figure 1). The patient also sustained nonoperative thoracic spine fractures, a left scapular fracture, and bilateral renal and adrenal contusions. He was transferred to our trauma center and underwent an emergent bilateral anterolateral (clamshell) thoracotomy, given the cardiac herniation and ongoing blood volume output from the left chest tube $(>200 \mathrm{cc} / \mathrm{h}$ with $1.5 \mathrm{~L}$ of total blood loss).

The right sided pleuropericardial defect extended adjacent to the phrenic nerve from the superior vena cava hiatus to the inferior vena cava hiatus (Figure 2 and Video 1). The heart was evaluated and found to have no contusions or

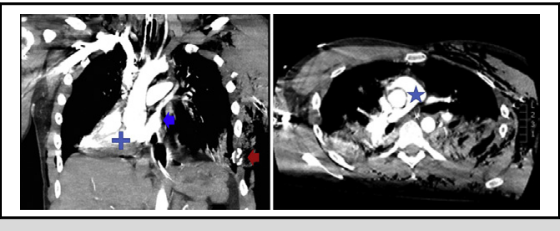

Chest CTA shows major blunt force trauma to the chest after a high-speed MVC.

CENTRAL MESSAGE

A rare case presentation of rightsided cardiac herniation from pericardial rupture following an MVC that required volume resuscitation, early operative intervention, and pericardial patch repair.

See Commentaries on pages 378 and 380 .

lacerations. It was manually reduced with an immediate improvement in hemodynamics. The pericardial defect was under tension when the edges were approximated. Thus, the defect was repaired using a nonabsorbable Bard Ventrio polypropylene (DAVOL INC, a subsidiary of C. R. Bard, Inc, Warwick, RI) and expanded polytetrafluoroethylene mesh prosthesis using polypropylene sutures in a loose interrupted fashion (Figure 3 ) to reduce the risk of constriction from fluid and edema.

An intraoperative transesophageal echocardiogram was performed that confirmed no additional cardiac injuries. No intraoperative or postoperative dysrhythmias were appreciated after cardiac reduction and pericardial repair. Intercostal bleeding from the left-sided rib fractures was controlled and a left lung laceration was repaired. He was transferred to the intensive care unit (ICU) for further clinical stabilization and cardiac monitoring. Postoperative day 1 troponin and creatine kinase levels peaked at $428 \mathrm{ng} / \mathrm{L}$ and $7588 \mathrm{U} / \mathrm{L}$, respectively, and decreased to normal within a few days of serial monitoring. A follow-up postoperative transesophageal echocardiogram remained unremarkable. He returned to surgery 3 days later for open reduction and internal fixation of his significant left-sided flail chest. After a prolonged ICU stay, the patient made a full recovery and 

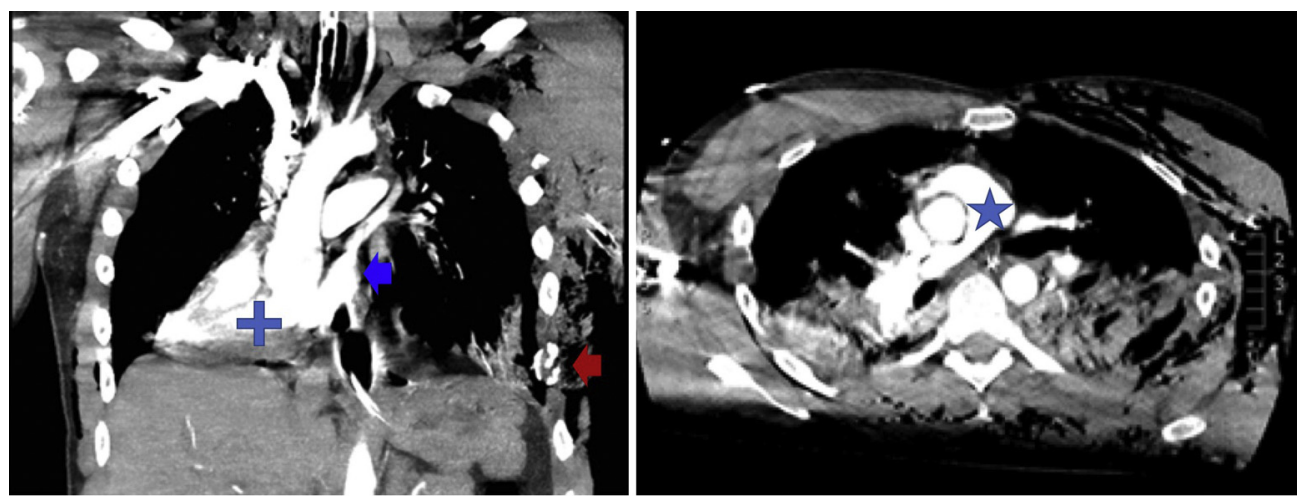

FIGURE 1. Chest computed tomography angiogram of a 24-year-old male patient who experienced major blunt force trauma to the chest following a highspeed motor vehicle collision. It demonstrates a flail chest (red arrow), narrowed pulmonary veins (blue arrow), cardiac torsion (blue cross), and pulmonary artery torsion (blue star) around the ascending aorta.

was discharged home. Informed patient consent was obtained for this report.

\section{DISCUSSION}

Pericardial rupture with cardiac herniation is a rare phenomenon following blunt traumatic injury-approximately $0.4 \%$. ${ }^{1}$ This complex injury is often fatal and typically discovered postmortem. Mortality rates for patients receiving hospital care range from $57 \%$ to $64 \%$. ${ }^{1}$ Pericardial rupture should be suspected in any patient with severe blunt chest trauma. ${ }^{2}$ Morel-Lavallee described a murmur in 1864 sounding like a splashing mill-wheel ("bruit de moulin") caused by hemopneumopericardium ${ }^{3}$ - difficult to assess in a trauma room setting. Findings on chest radiograph include pneumopericardium, pneumomediastinum, prominent cardiac silhouette, or new dextrocardia. ${ }^{1}$ If clinical stability permits, a computed tomography scan of the chest should be expeditiously obtained to diagnose

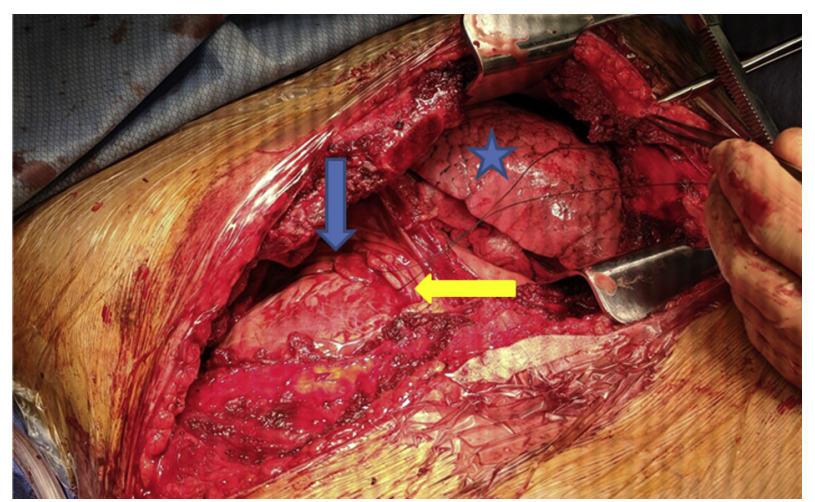

FIGURE 2. Intraoperative picture of a 24-year-old male patient following a motor vehicle collision. The patient sustained a right-sided pleuropericardial rupture (yellow arrow) with cardiac herniation into the right chest (blue arrow). The silk suture is retracting the pericardial defect. The left lung (blue star) can be seen in the left chest. The patient's head is toward the top of the picture. pericardial rupture and cardiac herniation as other trauma room adjuncts (focused assessment with sonography for trauma examination) may be limited and could potentially delay operative intervention. ${ }^{4}$ Pericardial rupture typically occurs along the pleural pericardium near the phrenic nerve $^{4}$ on the left side $(64 \%)$, followed by diaphragm $(18 \%)$, right-side $(9 \%)$, and superior mediastinum $(9 \%)$. Pleuropericardial rupture may cause heart herniation into the pleural space, causing heart constriction, strangulation, or torsion of the great vessels. Prompt surgical exploration to reposition the heart and correct the pericardial defect is the treatment of choice and may yield favorable outcomes. ${ }^{4}$ The operative approach is governed by the clinical scenario. Pericardial rupture and cardiac herniation may be addressed via a sternotomy or a left-sided thoracotomy. Hemodynamically unstable patients require a left-sided resuscitative thoracotomy with potential extension into a bilateral (clamshell) thoracosternotomy. Pericardial closure may be

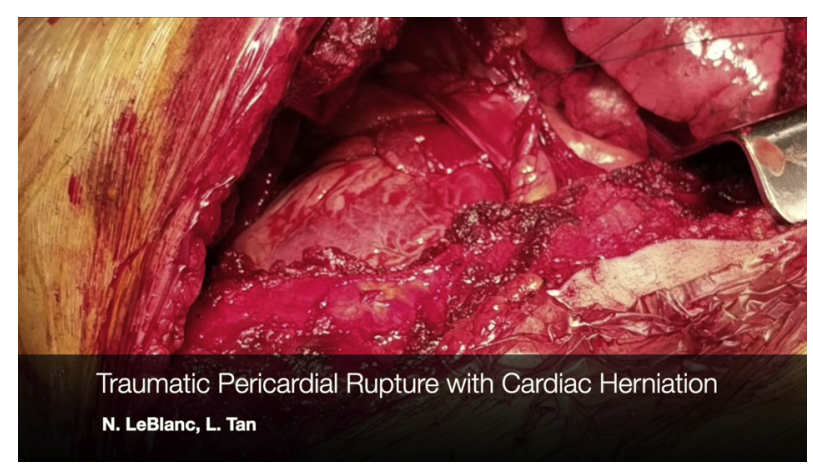

VIDEO 1. Intraoperative video of a 24 -year-old male patient following a high-speed motor vehicle collision. The patient sustained a right-sided pleuropericardial rupture with cardiac herniation into the right chest. The heart is visualized contracting in the right chest. The silk suture is retracting the pericardial defect. Video available at: https://www.jtcvs.org/article/ S2666-2507(20)30386-2/fulltext. 


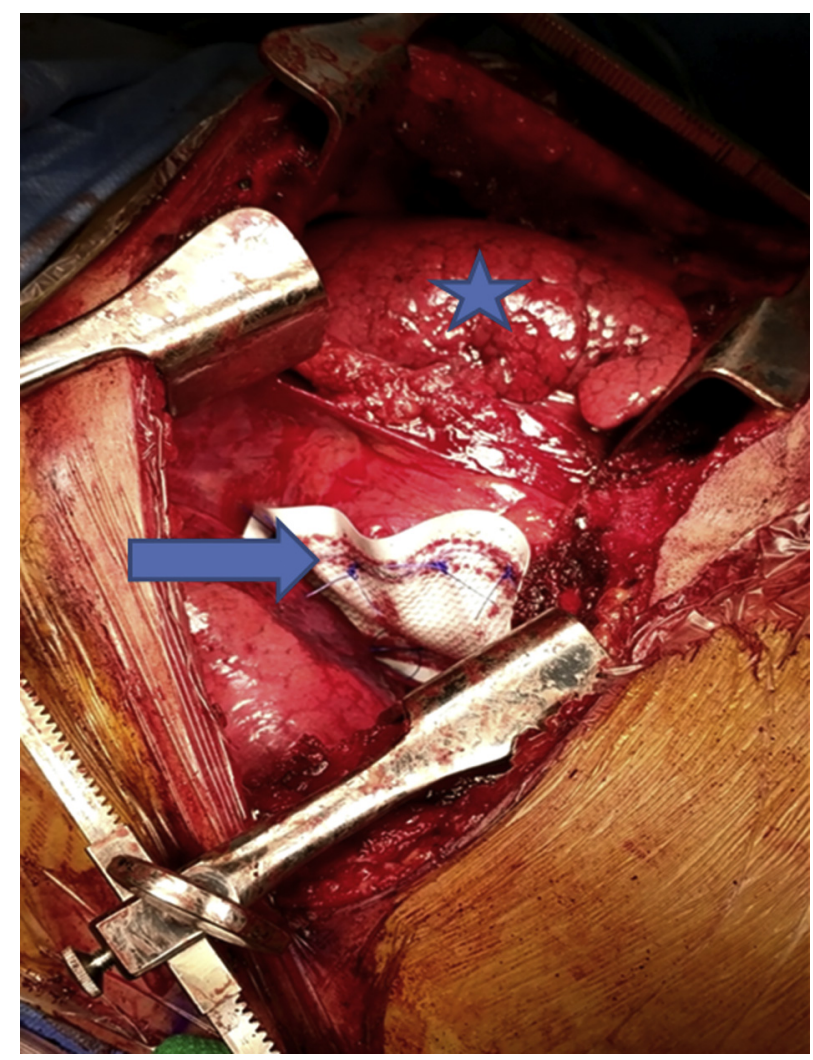

FIGURE 3. Intraoperative picture of a 24 -year-old male patient following a motor vehicle collision with mesh prosthesis repair of his right-sided pleuropericardial rupture (blue arrow). The left lung (blue star) can be seen in the left chest. performed with direct suturing or insertion of a patch if cardiac dilation or edema is a concern. ${ }^{5}$ Concomitant myocardial contusion is frequently encountered with these injuries and increases the risk of cardiac failure. ${ }^{5}$ ICU admission with telemetry and close clinical monitoring is warranted.

\section{References}

1. Sherren PB, Galloway R, Healy M. Blunt traumatic pericardial rupture and cardiac herniation with a penetrating twist: two case reports. Scand J Trauma Resusc Emerg Med. 2009; 17:64.

2. Janson JT, Harris DG, Pretorius J, Rossouw GJ. Pericardial rupture and cardiac herniation after blunt chest trauma. Ann Thorac Surg. 2003;75:581-2.

3. Fulda G, Brathwaite CE, Rodriguez A, Turney SZ, Dunham CM, Cowley RA. Blunt traumatic rupture of the heart and pericardium: a ten-year experience (1979-1989). J Trauma. 1991;31:167-73.

4. Dato GM, Arslanian A, Filosso PL, Aidala E, Adduci M, Bardi G, et al. Heart herniation after blunt chest trauma. J Thorac Cardiovasc Surg. 2002;123:367-8.

5. Lindenmann J, Matzi V, Neuboeck N, Porubsky C, Ratzenhofer B, Maier A, et al. Traumatic pericardial rupture with cardiac herniation. Ann Thorac Surg. 2010;89: 2028-30. 